\title{
RAFT Method for Preparation of Molecularly Imprinted Polymer-SBA15 Nanocomposite as a Pipette Tip Solid- Phase Microextraction Adsorbent
}

\author{
Marzieh Piryaei $^{1, *}(\mathbb{D})$, Mir Mahdi Abolghasemi ${ }^{1}$, Sayna Jodatvand ${ }^{1}$ \\ 1 Department of Chemistry, Faculty of Science, University of Maragheh, Maragheh, Iran \\ * Correspondence: m.piriyaei@gmail.com (M.P.);
}

Scopus Author ID 54389892400

Received: 10.01.2021; Revised: 5.02.2021; Accepted: 8.02.2021; Published: 13.02.2021

\begin{abstract}
Methoxyfenozide-selective surface molecularly imprinted polymer (MIP) sorbent was synthesized by reversible addition-fragmentation chain transfer (RAFT) polymerization based on support matrix of SBA-15. The size, morphology, composition, and properties of the prepared nanocomposite have also been characterized and determined using scanning electron microscopy (SEM) and Fourier-transform- infrared (FT-IR) spectroscopy. A miniaturized solid-phase extraction procedure has been developed for ultra-trace determination of methoxyfenozide adsorbed on polymethacrylic acid-functionalized Santa Barbara Amorphous type material (SBA-15), which is packed into a pipette- tip (PT). The PT-SPE was assembled by packing $2.0 \mathrm{mg}$ of nanocomposite of MIP as sorbent into a $100 \mu \mathrm{L}$ pipette tip. The method was optimized for several important extraction parameters such as sample volume, $\mathrm{pH}$, amount of sorbent, and eluent solvent. The results determine that the prepared adsorbent has a high extraction power. High sensitivity, non-use of organic solvent, high selectivity, and low detection limits are the unique advantages of the proposed method.
\end{abstract}

Keywords: reversible addition-fragmentation chain transfer polymerization; surface molecularly imprinted polymer (MIP); solid phase extraction; methoxyfenozide; SBA-15.

(C) 2021 by the authors. This article is an open-access article distributed under the terms and conditions of the Creative Commons Attribution (CC BY) license (https://creativecommons.org/licenses/by/4.0/).

\section{Introduction}

Molecular imprinting is defined as the accumulation of a network polymer matrix around a template molecule. It is a blend of molecular imprinting reactions consisting of template molecules, functional monomers, cross-linking monomers, polymerization initiators, and solvent solutions. Molecular imprinting polymers are obtained from the copolymerization reaction between the functional monomer and the transverse binder in the presence of a template molecule as a three-dimensional polymeric network with a strong transverse bond.Once formed, the template molecules are separated from the resulting structure, leading to the formation of bonds with the shape, size, and functional groups of the template. This concept was first introduced to silica in 1931 [1], while most works in history have focused on molecular imprinting in organic molecules.MIP has the same function as antibodies to enzymes. This is why they are designated as mimic antibodies. This could be explained as these polymers try to mimic structures like their natural counterparts [2]. In recent years, the use of MIP has been investigated in many fields of separation sciences such as capillary electrophoresis [3], crystal chromatography, solid-phase extraction [4, 5], and solid-phase microextraction [6]. The most common application of MIP in the pre-preparation process is in 
the solid-phase extraction [7]. Recently, it has been reported that the selectivity of solid-phase extraction (SPE) can be improved by employing molecular imprinting polymers or engraved molecular polymers because the identified sites of synthesis and the high selectivity of the engraved molecular polymers have made this feature obvious. Therefore, solid-phase extraction based on molecular imprinting polymers is one of the most successful and valuable applications. This method combines both SPE and MIP methods' advantages and displays high extraction efficiency, reusability, and good selectivity [8-27]. Pipette-tip extraction is a simpler and faster method of solid-phase extraction, introduced in 1998 as a new SPE tool. The solid phase absorber is placed inside the pipette and between the two filters. Due to the combination of the static phase (adsorbent) with the sample, several conditional steps required in the conventional SPE method are eliminated. After passing through the stationary phase, the sample solution is transferred to the waste container, and the stationary phase is washed with only $1 \mathrm{ml}$ of aqueous solution or buffer. After the washing step, the solvent is transferred to the waste container, the analyte absorbed by $0.1-0.3 \mathrm{ml}$ of its special solvent is injected into the GC or HPLC device, and the required analysis is performed. The time-consuming stage of compaction in the device is also eliminated due to the low volume of solvent used. Solid-phase extraction method - Pipette tip includes advantages such as faster extraction time (1-2 minutes), an extraction method for all analyzes, clean extraction, low sample volume (about 200 microliters) compared to the conventional SPE method, low solvent volume (200-400 microliters) and reduced solvent volume is wasted [28]. Although controlling the pathogen (using pesticides) in the agricultural sector is beneficial, pesticides cause disease and death in humans, and these problems are caused by different conditions of direct and indirect human contact with pesticides. As soon as human skin is exposed to the toxin, the toxin may be absorbed into the body or remain only on the skin's surface. The general effects of the condition on the skin are problems such as dermatitis (skin allergies). The absorption of pesticides in the body can cause problems for human health: such as eye irritation, in the case of further absorption of respiratory problems with systemic poisoning, which may eventually lead to death. Indirect moss with pesticides is caused by eating foods that have been infested with pesticides and can increase toxins in the human body, which are usually dependent on longterm exposure to these pesticides, which can lead to disease or not. Sunscreens work by increasing the body's speed of different activities, increasing them (for example, increased heart rate, sweating), or reducing them (complete cessation of breathing). For example, experimental populations with Malathion insect pesticides can cause sweating based on experience; this result is due to a series of physiological events in the body that respond to chemicals in the body and initially cause the enzyme's biochemical inactivity [29, 30]. Depending on the specific biochemical mechanism of an activity, a toxin may have very extensive effects on the body, or it may cause very limited changes in physiological activity in a specific area of the body or limbs. Malathion toxin simply inactivates an enzyme that is the interface between nerves. An enzyme that is inactivated by the parathyroid toxin or other pesticides is common in the body and, therefore, has various effects on most body systems and sweating [31].Methoxyfenoside (N-tert-butyl-N '- (3-methoxy-o-toluoy) -3, 5-xylohydrazide), a member of the dia-cylhydrazine family, is an insecticide. It is a white powder with the chemical formula $\mathrm{C}_{22} \mathrm{H}_{28} \mathrm{~N}_{2} \mathrm{O}_{3}$, which acts as ecdysone and active at the beginning of consumption. Methoxyphenoside is emitted when $\mathrm{NO}_{2}$ toxic gases evaporate or decompose. It is active in all stages of feeding the lichens and turning them into butterflies, thus interrupting the feeding prematurely, the lichens' deadly peeling, and being an active, fat-soluble substance. It is also 
an active ingredient in fat.In the present study, the molecular imprinting polymers synthesized by RAFT polymerization have been activated on the SBA-15 surface, and as an adsorbent in the pipette tip-SPE, it has been used to extract methoxyfenozide toxin from the aqueous sample.

\section{Materials and Methods}

\subsection{Materials.}

All materials including methacrylic acid monomer (MAA), ethylene glycol dimethacrylate (EGDMA), azobis (2-methylpropionitrile) (AIBN), carbon disulfide, bromine benzene, magnesium, SBA-15, chloromethylcetyl, Sulfuric acid, tetrahydrofuran, methanol, and acetone were produced by Lubacam and Merck. The methoxy-phenoxide toxin was prepared from Dow Agro Sciences.

\subsection{Apparatus.}

In this study, to analyze the data, the high-performance liquid chromatographic device of the German model KNAUER Smartline, equipped with a four-solvent pump, manual injection with a loop volume of $20 \mu \mathrm{L}$, with a column filled with polymer C18 (240 x $0.6 \mathrm{~mm})$ and the UV-Vis detector (model SV-2600UV) was used in the adjustable wavelength range from 200 to $400 \mathrm{~nm}$. To wash the column, the flow rate started from $0.2 \mathrm{~mL} / \mathrm{min}$, and as this flow rate was increasing, it reached 0.5 and then 0.7 , and finally, the flow rate was adjusted to $1 \mathrm{~mL} / \mathrm{min}$. Samples extracted in the same volume, through column $\mathrm{C}_{18}$, were passed by the combined mobile methanol/acetonitrile phase with a volume ratio of 70:30 at a temperature of $20{ }^{\circ} \mathrm{C}$ and a flow rate of $1 \mathrm{~mL}$ per minute, and a wavelength of $240 \mathrm{~nm}$ measured by UV-Vis detector. The injection volume was $20 \mu \mathrm{L}$ and the time required for the peaks to appear was 5 minutes. Cromgate software was used to record the chromatogram and measure the area under the peak.

Material morphology was recorded using a scanning electron microscope of the LEO 1430 VP model. To further prove the synthesized material's synthesis and chemical structure, a spectroscopic Bruker 27 infrared spectroscope made in Germany was used.

\subsection{Synthesis of SBA-15-Cl.}

To synthesize SBA-15-Cl [31,32], approximately $250 \mathrm{mg}$ of SBA-15 was initially activated by $3 \mathrm{Mm}$ hydrochloric acid so that $100 \mathrm{~mL}$ was added to $0.5 \mathrm{~g}$ SBA-15 and sub. The reflux device was placed after 24 hours of reaction; the product was washed with distilled water and dried. About $250 \mathrm{~mL}$ of activated SBA-15 was poured into about $2 \mathrm{~mL}$ (4-chloromethyl) phenylephrine methoxy silane and $10 \mathrm{~mL}$ of dry toluene solvent into a $100 \mathrm{~mL}$ balloon, and then, the temperature reached $90{ }^{\circ} \mathrm{C}$ and was subjected to nitrogen gas flow. After 24 hours, the mixed particles were separated by filter paper and dried in an oven under a vacuum atmosphere.

\subsection{Synthesis of the transfer agent to the increase-detachable radical polymerization chain} reversible on the SBA-15-Cl surface.

The phenyl magnesium bromide solution ( $1 \mathrm{~mol}$ in dry tetrahydrofuran) was first synthesized by the Grignard reaction, as follows: about $17 \mathrm{~mL}$ of dry tetrahydrofuran solvent, about $0.5 \mathrm{~g}$ of magnesium fragments, and $1.2 \mathrm{~mL}$ of bromobenzene in droplets inside the 
balloon. Two drops were poured under reflux, and a tip of the spatula was added from the solid iodine after 24 hours of reaction at $70^{\circ} \mathrm{C}$, on the reaction solution $1 \mathrm{~mL}$ of carbon disulfide for 10 minutes and in the form of drops under $40{ }^{\circ} \mathrm{C}$ was added, and after 1 hour of the above reaction, $500 \mathrm{mg}$ of the prepared SBA-15-Cl powder synthesized in previous sections was added to it. The reaction lasted for 60 hours at $70{ }^{\circ} \mathrm{C}$ and under the influence of nitrogen gas. After 60 hours, $25 \mathrm{~mL}$ of the molar hydrochloric acid solution was added to the reaction mixture. Then the product was rinsed several times with tetrahydrofuran, methanol, and acetone solvents. The resulting SBA-15-RAFT was dried at $40{ }^{\circ} \mathrm{C}$ for 24 hours in a vacuum atmosphere.

2.5. Methoxyfenoside polymer template synthesis on SBA-15-RAFT (SBA-15-MIP nanocomposite).

For sealing from SBA-15 surface, SBA-15-RAFT powder (modified with dithioesters compound) obtained from previous stages in a solution of $0.4 \mathrm{mmol}(0.033 \mathrm{~mL})$ methacrylic monomer acid and $0.1 \mathrm{mM}(0.16 \mathrm{~g})$ of methoxyfenozide toxin in $10 \mathrm{~mL}$ of methanol was subjected to nitrogen atmospheres to penetrate the monomer and template molecules into the SAB-15 cavities. After a few minutes, $200 \mathrm{mg}$ of AIBN and $2 \mathrm{mM}(0.377 \mathrm{~mL})$ of ethylene glycol di methacrylate cross-linker were added to the monomer and template mixture and at $60^{\circ} \mathrm{C}$ temperature. The oil baths and reflux devices were installed to perform the polymerization process. After 20 hours, the solution was centrifuged in a test tube. The resulting sediment was washed several times with a volumetric composition of water and methanol (5: 1) and dried at room temperature.

\subsection{Preparation of standard solutions.}

The stock solution was prepared at a concentration of $100 \mathrm{ng} / \mathrm{mL}$ by dissolving 0.0422 $\mathrm{g}$ of methoxyfenozide poison in $10 \mathrm{~mL}$ of methanol. The working solutions were prepared by adding the necessary amounts of water daily so that for each analysis, $10 \mathrm{~mL}$ of mother solution was used with the final concentration of $1 \mathrm{ng} / \mathrm{mL}$ prepared in the analysis solution (water). Methoxyfenoside was kept in the dark at $4^{\circ} \mathrm{C}$ to prevent the destruction of the toxin.

\subsection{Extraction process with the solid phase-pipette tip.}

The extraction process was carried out with a solid-pipette tip phase with $100 \mu \mathrm{m}$ microslippers connected to an insulin syringe. First, the microspores were washed with $1 \mathrm{~mL}$ of methanol and $1 \mathrm{~mL}$ of deionized water and dried at room temperature to prepare for the extraction process. The two sides of the micro-spasm were covered with cotton to prevent the absorption from being wasted; $1 \mathrm{mg}$ of the synthesized adsorbent was poured into the microspasm so that it was placed exactly between the two pieces of cotton. To activate the adsorbent powder with $1 \mathrm{~mL}$ of methanol solution: water $(9: 1 \mathrm{~mL})$ was washed. $10 \mathrm{~mL}$ of the analytical solution was extracted by a syringe attached to a micro-sampler and finally dissolved in one $\mathrm{ml}$ of soluble methanol solution, which was stored in brown glass containers for injection into the HPLC-UV device. 


\section{Results and Discussion}

3.1. Investigation of the morphological morphology of the synthesized molecular template on SBA-15 mesoporous (SBA-15 -MIP nanocomposite).

In research on nanostructured materials' properties, the electron microscope is one of the most important and widely used devices. Most studies on the properties of nanostructured materials use scanning electron microscopy to determine their size and shape.

Figure 1 shows an SEM image for SBA-15. This image shows short rod-shaped structures with a uniform size of about one micrometer of pure SBA-15. However, in Figure 2, which displays the modified SBA-15 image with the polymer of template, it can be seen that the SBA-15 surface is more uneven. Moreover, the formation of a thin polymer layer of the molecular template is destroyed.

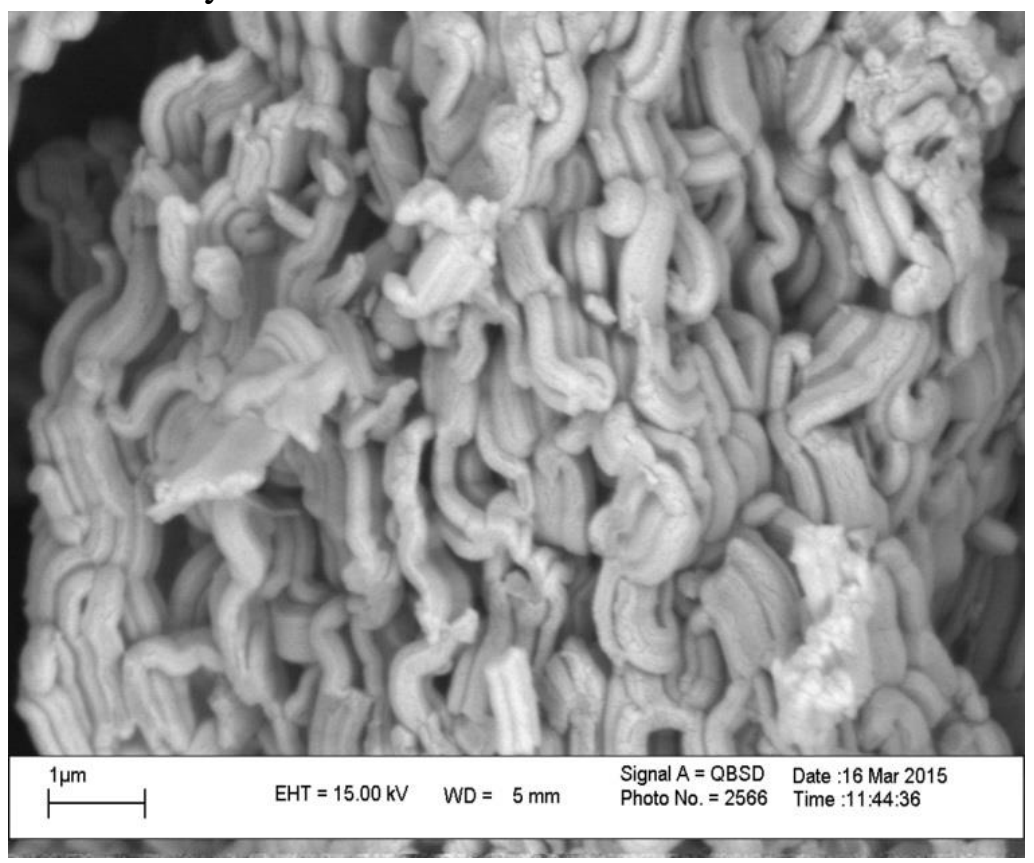

Figure 1. SEM images related to SBA-15.

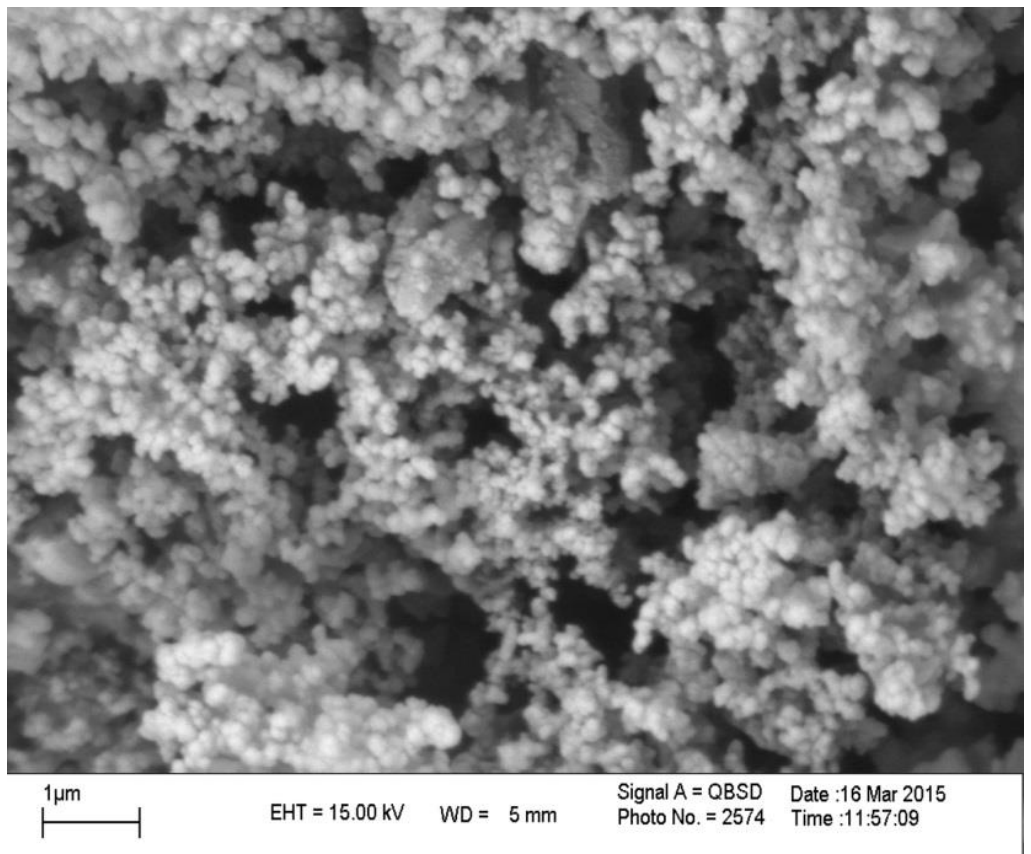

Figure 2. SEM image related to SBA-15 modified by methotrexate polymer molecular form. 


\subsection{Examination of pure FT-IR SBA-15 spectrum and SBA-15-MIP nanocomposite.}

In the FT-IR spectrum obtained from pure SBA-15 analysis, the peaks observed in 3427 and $1626 \mathrm{~cm}^{-1}$ are related to the vibration of $-\mathrm{OH}$ groups due to water absorbed by mesoporous and SBA-15 hydroxyl groups, and also $1079 \mathrm{~cm}^{-1}$ peaks are related to Si-O-Si asymmetric tensile vibrations, and $849 \mathrm{~cm}^{-1}$ region peak peaks are related to $\mathrm{Si}-\mathrm{O}-\mathrm{Si}$ symmetric tensile vibrations. Si-O-Si bending vibrations are also observed in the $471 \mathrm{~cm}^{-1}$ region.

In the FT-IR spectrum obtained from SBA-15-MIP nanocomposite, the peaks observed in the $1731 \mathrm{~cm}^{-1}$ area are related to the tensile vibrations of the carbonyl groups in EGDMA and MAA. Furthermore, the peak for C-Cl vibrations, which should be in the $690 \mathrm{~cm}^{-1}$ area, is not visible, which indicates the reaction of the peak's gasoline disappearance and disappearance and the conversion of SBA-15-Cl to SBA-15-RAFT and instead the peak related to the tensile vibrations of the $\mathrm{C}=\mathrm{S}$ bond can be seen in the $1443 \mathrm{~cm}^{-1}$ area. The bending vibrations associated with the $\mathrm{C}=\mathrm{C}$ bond are also easily visible in the $1498 \mathrm{~cm}^{-1}$ region. These observations prove the formation of SBA-15-MIP nanocomposites. Figure 3 displays the SBA15 FT-IR spectrum and the SBA-15-MIP nanocomposite.

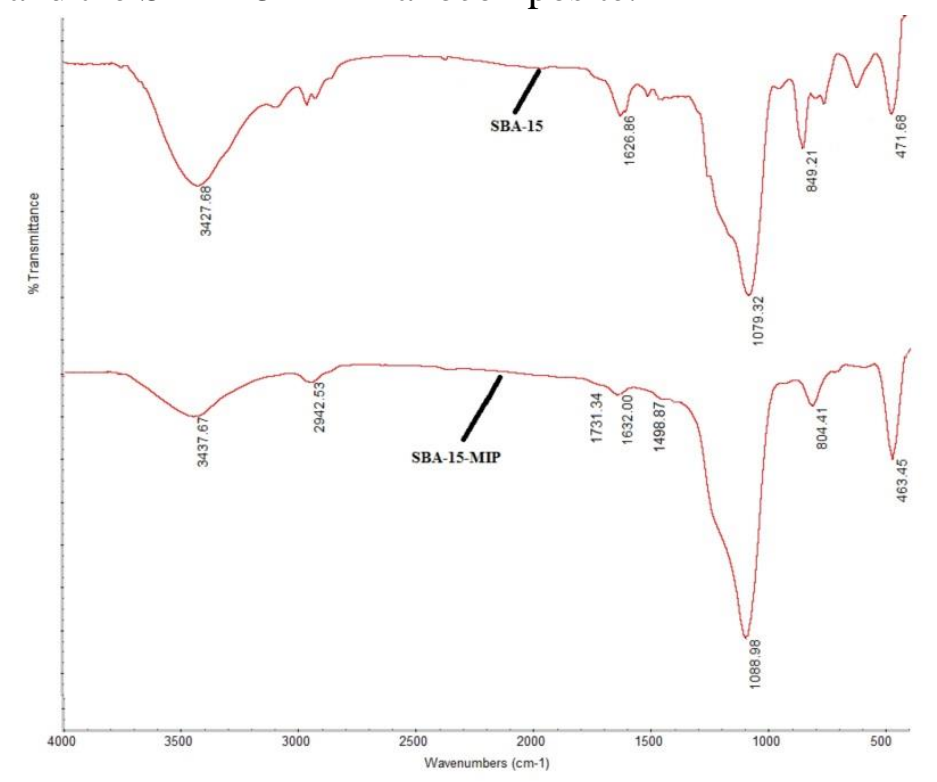

Figure 3. FT-IR spectrum for pure SBA-15 and SBA-15-MIP nanocomposite.

\subsection{Optimization of extraction method with solid phase-pipette tip.}

To test the strength and efficiency of SBA-15-MIP nanocomposite in the solid phase extraction process, a toxic pipette tip of methoxyfenozide was employed as a test solution. The solid-phase-pipette extraction method is usually preferred since this miniature method coupled with HPLC-UV chromatography is a quick way to estimate contaminants. If the adsorbent is a molecular template polymer, the extraction is completely selective and without disturbing agents' absorption. Compared to conventional cartridge extraction methods with a solid phase, the pipette's tip has a special conical shape with different diameters in the two end parts, which makes it a desirable cartridge. Placing a small amount of adsorbent at the end of the pipette tip provides enough distance to pass the sample solution, while the other end with a larger diameter is suitable for easy sampling. In this study, the cartridge consists of compressing about one mg of the adsorbent inside a 100-microliter pipette, which has a high absorption capacity due to the unique structure of SBA-15 and its high surface area. Several parameters were optimized 
in the conducted work to obtain satisfactory extraction results, including the amount of hot adsorbent, $\mathrm{pH}$, and sample solution volume, tip, and volume of solvent and adsorbent.

\subsection{The amount of adsorbent.}

The amount of compressed adsorbent in the cartridge is a key factor in this study. The amount of adsorbent was checked from one to three $\mathrm{mg}$, considering the low consumption. The best results were observed in one $\mathrm{mg}$. With increasing the adsorbent value to two and three $\mathrm{mg}$, a slight improvement was observed in the extraction conditions. Therefore, one mg was selected as the optimal parameter. Figure 4 displays the effect of the amount of adsorbent on the extraction efficiency.

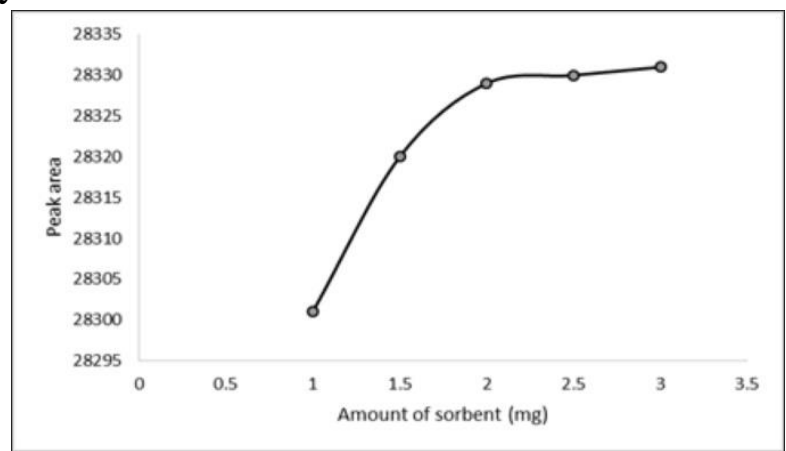

Figure 4. Chart analyte peak area changes according to changes in the amount of adsorbent.

\section{5. $p H$ effect.}

The sample solution's pH indicates how stable, molecular, or dissociated it is from the ionite ion. Since the sample solution's $\mathrm{pH}$ is one of the significant factors in transferring the analysis from the sample solution to the adsorbent present in the pipette tip, the effect of $\mathrm{pH}$ was increased by adding $\mathrm{HCl}$ or $\mathrm{NaOH}$ in the range of 2-10. The highest extraction efficiency was obtained at $\mathrm{pH}=10$, which is presumably due to the $\mathrm{pH}$; in this $\mathrm{pH}$, methoxyfenozide remains molecularly in the environment, so its transfer to the adsorbent at this $\mathrm{pH}$ is better. Figure 5 displays the $\mathrm{pH}$ effect diagram on the extraction efficiency.

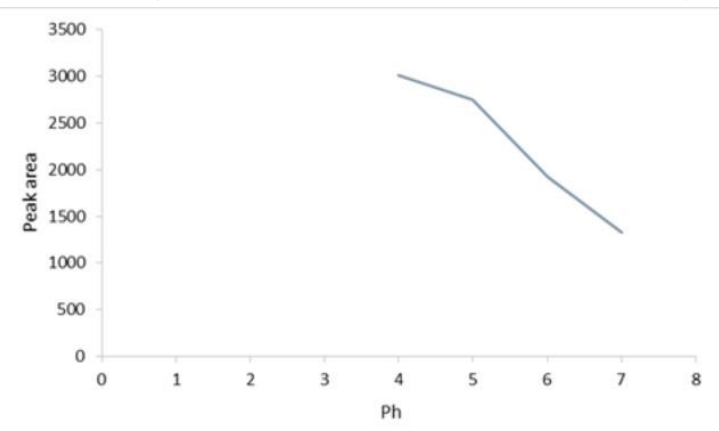

Figure 5. Chart analyte peak area changes according to changes in $\mathrm{pH}$.

\subsection{Analytical solution volume.}

It is necessary to optimize the analysis volume in this method to obtain reliable analysis results and confirm the method's sensitivity. Accordingly, different volumes of 5-20 mL were examined; the analysis volume was selected according to the acceptable time in all stages of the method and the required sensitivity. Hence, according to the diagram, the sample size of 10 $\mathrm{mL}$ was considered the optimal volume for low extraction time and reliable sensitivity in the process. Figure 6 displays the effect of the analysis volume on extraction efficiency. 


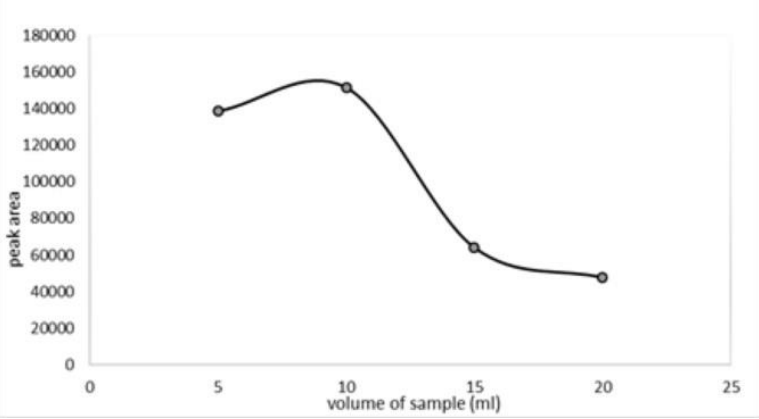

Figure 6. Chart analyte peak area changes according to the changes of analytes.

\subsection{The type of adsorbent solvent.}

Another factor that alters the efficiency of extraction is the type of adsorbent solvent. Depending on the type of analysis, different solvents are used for separation. There are several points in choosing a solvent and absorbable solvent, including solvent and adsorbent compatibility with the desired measurement method, the purity of solvent and adsorption purity, inexpensiveness, and non-toxicity. After extraction under the same conditions, three solvents of methanol, hexane, and acetonitrile were used in the adsorption phase to investigate this factor. After recording the adsorbent solutions' chromatograms, the results were compared concerning the number of extracted species and their amount, respectively. The results revealed that the lowest level of the chromatogram compounds' peaks was associated with the time when hexane was used as the soluble and absorbable solvent. In contrast, maximum efficiency and adsorption were obtained using methanol solvent. Figure 7 displays the effect of solvent type the extraction efficiency.

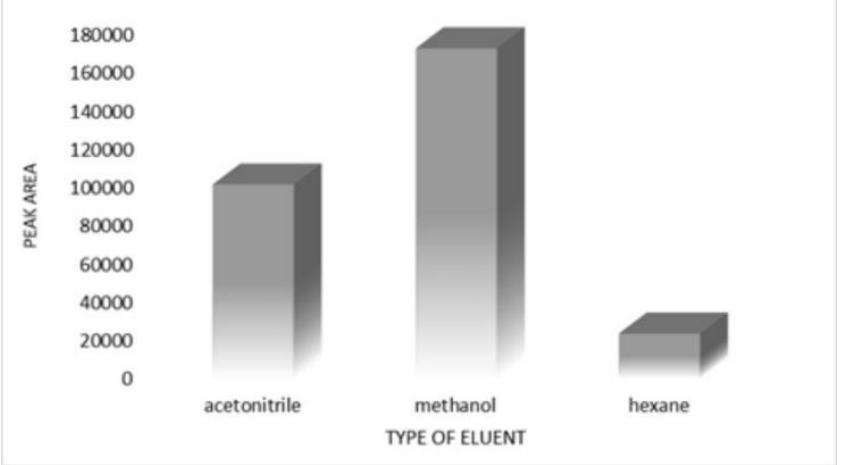

Figure 7. Chart analyte peak area changes according to changes in desorption solvent.

\subsection{Quantitative assessments and analysis of real samples.}

The extraction method with a solid phase-pipette tip was evaluated under optimal conditions by testing the analytical parameters of methoxyfenozide poison in the sample. The results of the study are given in Table 1 . This indicates that the fiber has good repeatability. Compared to other coating materials in literature data such as PDMS for extraction and determination of methoxyfenozide, the proposed fiber shows a suitable LOD.

For the toxin in question, by absorbing the polymer, the molecular template on the surface of the mesoporous SBA-15 was calculated based on the signal-to-noise ratio of $3=$ $0.004 \mathrm{mg} / \mathrm{mL}$ (Table 1). The linear range of this method was tested by extracting different water standards by increasing the concentration. With the desired adsorbent, this method obtained a valid linear range with a correlation coefficient of 0.999 . The method's accuracy 
was determined by repeated extraction of three folds of aqueous solutions with a concentration of $1 \mathrm{mg} / \mathrm{L}$ under optimal conditions. The relative standard deviation using the molecular template's polymer adsorption was $1.52 \%$, which indicates that the adsorbent has valid repeatability.

Table 1. Comparison of the proposed method with other methods of Methoxyfenozide extraction [32], [33] Liquid Chromatography-Tandem Mass Spectrometry (LC-MS/MS), and [34] dispersive liquid-liquid microextraction.

\begin{tabular}{|c|c|c|c|c|c|c|c|c|c|c|}
\hline \multirow[t]{2}{*}{ Compound } & \multicolumn{4}{|c|}{ Current method } & \multicolumn{2}{|c|}{ QuEChERS } & \multicolumn{2}{|c|}{$\begin{array}{c}\text { Liquid } \\
\text { Chromatography- } \\
\text { Tandem Mass } \\
\text { Spectrometry (LC- } \\
\text { MS/MS) }\end{array}$} & \multicolumn{2}{|c|}{$\begin{array}{c}\text { Dispersive liquid- } \\
\text { liquid } \\
\text { microextraction }\end{array}$} \\
\hline & $\mathrm{DLR}^{\mathrm{a}}$ & $\left(\mathrm{R}^{2}\right)^{\mathrm{b}}$ & $\mathrm{LOD}^{\mathrm{c}}$ & $\% \mathrm{RSD}^{\mathrm{d}}$ & LOD & $\% \mathrm{RSD}$ & LOD & $\%$ RSD & LOD & $\% \mathrm{RSD}$ \\
\hline Methoxyfenozide & $\begin{array}{c}0.01- \\
100\end{array}$ & 0.999 & 0.004 & 8.2 & 0.005 & $<20$ & 0.005 & - & 0.0025 & 4.1 \\
\hline
\end{tabular}

${ }^{\mathrm{a}}$ Dynamic Linear Rang $\left(\mu \mathrm{gml}^{-1}\right)$;

${ }^{\mathrm{b}}$ Regression coefficient;

'Limit of detection calculated as three times the baseline noise $\left(\mathrm{ngml}^{-1}\right)$;

${ }^{\mathrm{d}}$ Relative Standard Deviation.

\section{Conclusions}

In these studies, a highly sensitive method has been developed based on solid-phase extraction, a pipette tip based on the use of a molecular imprinting polymer adsorbent on the SBA-15 surface to determine the toxicity of methoxyfenozide in aqueous samples. The used method maintains a valid linear range, repeatability, and high sensitivity. The results determine that the prepared adsorbent has a high extraction power. High sensitivity, non-use of organic solvent, high selectivity, and low detection limits are the unique advantages of the proposed method.

\section{Funding}

This research received no external funding.

\section{Acknowledgments}

The authors declare no acknowledgments.

\section{Conflicts of Interest}

There is no conflict of interest to the authors.

\section{References}

1. Cheong, W.J.; Ali, F.; Choi, J.H.; Lee, J.O.; Yune Sung, K. Recent applications of molecular imprinted polymers for enantio-selective recognition. Talanta 2013, 106, 45-59, https://doi.org/10.1016/j.talanta.2012.11.049.

2. Nilsson, J.; Spégel, P.; Nilsson, S. Molecularly imprinted polymer formats for capillary electrochromatography. J. Chromatogr. B 2004, 804, 3-12, https://doi.org/10.1016/j.jchromb.2003.12.036.

3. Quaglia, M.; Sellergren, B.; De Lorenzi, E. Approaches to imprinted stationary phases for affinity capillary electrochromatography. J. Chromatogr. 2004, 1044, 53-66, https://doi.org/10.1016/j.chroma.2004.05.055.

4. Lanza, F.; Sellergren, B. Molecularly imprinted extraction materials for highly selective sample cleanup and analyte enrichment. Adv. Chromatogr. 2001, 41, 137-173, https://doi.org/10.1002/aic.1177910. 
5. Masqué, N.; Marcé, R.M.; Borrull, F. New polymeric and other types of sorbents for solid-phase extraction of polar organic micropollutants from environmental water. TrAC, Trends Anal. Chem. 1998, 17, 384-394, https://doi.org/10.1016/S0165-9936(98)00019-3.

6. Koster, E.H.M.; Crescenzi, C.; den Hoedt, W.; Ensing, K.; de Jong, G.J. Fibers Coated with Molecularly Imprinted Polymers for Solid-Phase Microextraction. Anal. Chem. 2001, 73, 3140-3145, https://doi.org/10.1021/ac001331x.

7. Celiz, M.D.; Aga, D.S.; Colón, L.A. Evaluation of a molecularly imprinted polymer for the isolation/enrichment of $\quad \beta$-estradiol. Microchem. $\quad J . \quad 2009, \quad 92, \quad 174-179$, https://doi.org/10.1016/j.microc.2009.03.007.

8. Zheng, M.-M.; Gong, R.; Zhao, X.; Feng, Y.-Q. Selective sample pretreatment by molecularly imprinted polymer monolith for the analysis of fluoroquinolones from milk samples. J. Chromatogr. 2010, 1217, 20752081, https://doi.org/10.1016/j.chroma.2010.02.011.

9. Gurnani, P.; Floyd, T.; Tanaka, J.; Stubbs, C.; Lester, D.; Sanchez-Cano, C.; Perrier, S. PCR-RAFT: rapid high throughput oxygen tolerant RAFT polymer synthesis in a biology laboratory. Polymer Chemistry 2020, 11, 1230-1236, https://doi.org/10.1039/C9PY01521C.

10. Xue, S.; Meng, F.; Zhang, P.; Bao, J.; Wang, J.; Zhao, K. Influence of water re-curing on microstructure of heat-damaged cement mortar characterized by low-field NMR and MIP. Construction and Building Materials 2020, 262, 120532, https://doi.org/10.1016/j.conbuildmat.2020.120532.

11. Rocha, L.B.; Soares, H.T.; Mendes, M.I.P.; Cabrita, A.; Schaberle, F.A.; Arnaut, L.G. Necrosis Depth and Photodynamic Threshold Dose with Redaporfin-PDT. Photochem. Photobiol. 2020, 96, 692-698, https://doi.org/10.1111/php.13256.

12. Becker, B.; Feng, X.; Yin, Y.; Holzinger, A. Desiccation tolerance in streptophyte algae and the algae to land plant transition: evolution of LEA and MIP protein families within the Viridiplantae. J. Exp. Bot. 2020, 71, 3270-3278, https://doi.org/10.1093/jxb/eraa105.

13. Abbasy, L.; Mohammadzadeh, A.; Hasanzadeh, M.; Razmi, N. Development of a reliable bioanalytical method based on prostate specific antigen trapping on the cavity of molecular imprinted polymer towards sensing of PSA using binding affinity of PSA-MIP receptor: A novel biosensor. J. Pharm. Biomed. Anal. 2020, 188, 113447, https://doi.org/10.1016/j.jpba.2020.113447.

14. Sun, Y.; Guo, Y.; Feng, X.; Jia, M.; Ai, N.; Dong, Y.; Zheng, Y.; Fu, L.; Yu, B.; Zhang, H.; Wu, J.; Yu, X.; Wu, H.; Kong, W. The behavioural and neuropathologic sexual dimorphism and absence of MIP-3 $\alpha$ in tau P301S mouse model of Alzheimer's disease. J. Neuroinflammation 2020, 17, 72, https://doi.org/10.1186/s12974-020-01749-w.

15. Mazouz, Z.; Mokni, M.; Fourati, N.; Zerrouki, C.; Barbault, F.; Seydou, M.; Kalfat, R.; Yaakoubi, N.; Omezzine, A.; Bouslema, A.; Othmane, A. Computational approach and electrochemical measurements for protein detection with MIP-based sensor. Biosensors Bioelectron. 2020, 151, 111978, https://doi.org/10.1016/j.bios.2019.111978.

16. Kou, Y.; Wu, T.; Zheng, H.; Kadasala, N.R.; Yang, S.; Guo, C.; Chen, L.; Liu, Y.; Yang, J. Recyclable Magnetic MIP-Based SERS Sensors for Selective, Sensitive, and Reliable Detection of Paclobutrazol Residues in Complex Environments. ACS Sustainable Chemistry \& Engineering 2020, 8, 14549-14556, https://doi.org/10.1021/acssuschemeng.0c05065.

17. Serrano, R.; Grindlay, G.; Niedzielski, P.; Gras, L.; Mora, J. Evaluation of MIP-OES as a detector in DLLME procedures: application to Cd determination in water samples. J. Anal. At. Spectrom. 2020, 35, 1351-1359, https://doi.org/10.1039/D0JA00113A.

18. Ciechanowska, A.; Popiolek-Barczyk, K.; Pawlik, K.; Ciapała, K.; Oggioni, M.; Mercurio, D.; De Simoni, M.-G.; Mika, J. Changes in macrophage inflammatory protein-1 (MIP-1) family members expression induced by traumatic brain injury in mice. Immunobiology 2020, 225, 151911, https://doi.org/10.1016/j.imbio.2020.151911.

19. Scheffler, M.; Neufeld, J.S.; Hölscher, M. An MIP-based heuristic solution approach for the locomotive assignment problem focussing on (dis-)connecting processes. Transportation Research Part B: Methodological 2020, 139, 64-80, https://doi.org/10.1016/j.trb.2020.05.020.

20. Pirzada, M.; Sehit, E.; Altintas, Z. Cancer biomarker detection in human serum samples using nanoparticle decorated epitope-mediated hybrid MIP. Biosensors Bioelectron. 2020, 166, 112464 , https://doi.org/10.1016/j.bios.2020.112464.

21. Kovalev, I.V.; Voroshilova, A.A. Overview of the II International Conference "MIP: Engineering-2020: Modernization, Innovations, Progress: Advanced Technologies in Material Science, Mechanical and 
Automation Engineering”. IOP Conference Series: Materials Science and Engineering 2020, 862, 011001, https://doi.org/10.1088/1757-899X/862/1/011001.

22. Adu, A.A.; Neolaka, Y.A.B.; Riwu, A.A.P.; Iqbal, M.; Darmokoesoemo, H.; Kusuma, H.S. Synthesis, characterization and evaluation of swelling ratio on magnetic p53-poly(MAA-co-EGDMA)@GO-Fe3O4 (MIP@GO-Fe3O4)-based p53 protein and graphene oxide from kusambi wood (Schleichera oleosa). Journal of Materials Research and Technology 2020, 9, 11060-11068, https://doi.org/10.1016/j.jmrt.2020.08.003.

23. Tancharoen, C.; Sukjee, W.; Yenchitsomanus, P.-t.; Panya, A.; Lieberzeit, P.A.; Sangma, C. Selectivity enhancement of MIP-composite sensor for explosive detection using DNT-dengue virus template: A coimprinting approach. Mater. Lett. 2021, 285, 129201, https://doi.org/10.1016/j.matlet.2020.129201.

24. Toscano, A.; Ferreira, D.; Morabito, R. Formulation and MIP-heuristics for the lot sizing and scheduling problem with temporal cleanings. Comput. Chem. Eng. 2020, 142, 107038, https://doi.org/10.1016/j.compchemeng.2020.107038.

25. Khosrokhavar, R.; Motaharian, A.; Milani Hosseini, M.R.; Mohammadsadegh, S. Screen-printed carbon electrode (SPCE) modified by molecularly imprinted polymer (MIP) nanoparticles and graphene nanosheets for determination of sertraline antidepressant drug. Microchem. J. 2020, 159, 105348, https://doi.org/10.1016/j.microc.2020.105348.

26. Piryaei, M.; Nazemiyeh, H. Preconcentration of digoxin using a synthetic imprinted polymer deposited upon the surface of double-layered hydroxides on porous anodised aluminium wire a triple solid-phase microextraction fibre. Phytochem. Anal. 2020, 31, 636-642, https://doi.org/10.1002/pca.2929.

27. Masqué, N.; Marcé, R.M.; Borrull, F. Molecularly imprinted polymers: new tailor-made materials for selective solid-phase extraction. TrAC, Trends Anal. Chem. 2001, 20, 477-486, https://doi.org/10.1016/S0165-9936(01)00062-0.

28. Mazloum-Ardakani, M.; Sheikh-Mohseni, M.A.; Abdollahi-Alibeik, M.; Benvidi, A. Electrochemical sensor for simultaneous determination of norepinephrine, paracetamol and folic acid by a nanostructured mesoporous material. Sensors Actuators B: Chem. 2012, 171-172, 380-386, https://doi.org/10.1016/j.snb.2012.04.071.

29. Xia, Y.; Zhi, X.; Wang, X.; Chen, M.; Cheng, J. Ultrasound-enhanced surfactant-assisted dispersive liquidliquid microextraction and high-performance liquid chromatography for determination of ketoconazole and econazole nitrate in human blood. Anal. Bioanal. Chem. 2012, 402, 1241-1247, https://doi.org/10.1007/s00216-011-5508-z.

30. Hansen, F.; Øiestad, E.L.; Pedersen-Bjergaard, S. Bioanalysis of pharmaceuticals using liquid-phase microextraction combined with liquid chromatography-mass spectrometry. J. Pharm. Biomed. Anal. 2020, 189, 113446, https://doi.org/10.1016/j.jpba.2020.113446.

31. Sereshti, H.; Semnani Jazani, S.; Nouri, N.; Shams, G. Dispersive liquid-liquid microextraction based on hydrophobic deep eutectic solvents: Application for tetracyclines monitoring in milk. Microchem. J. 2020, 158, 105269, https://doi.org/10.1016/j.microc.2020.105269.

32. Meng, M.; Meng, X.; Liu, Y.; Liu, Z.; Han, J.; Wang, Y.; Luo, M.; Chen, R.; Ni, L.; Yan, Y. An ion-imprinted functionalized SBA-15 adsorbent synthesized by surface imprinting technique via reversible additionfragmentation chain transfer polymerization for selective removal of $\mathrm{Ce}$ (III) from aqueous solution. $J$. Hazard. Mater. 2014, 278, 134-143, https://doi.org/10.1016/j.jhazmat.2014.06.002.

33. Li, S.; Gao, H.; Zhang, J.; Li, Y.; Peng, B.; Zhou, Z. Determination of insecticides in water using in situ halide exchange reaction-assisted ionic liquid dispersive liquid-liquid microextraction followed by highperformance liquid chromatography. J. Sep. Sci. 2011, 34, 3178-3185, https://doi.org/10.1002/jssc.201100577.

34. Martínez-Domínguez, G.; Nieto-García, A.J.; Romero-González, R.; Frenich, A.G. Application of QuEChERS based method for the determination of pesticides in nutraceutical products (Camellia sinensis) by liquid chromatography coupled to triple quadrupole tandem mass spectrometry. Food Chem. 2015, 177, 182-190, https://doi.org/10.1016/j.foodchem.2015.01.032. 